\title{
Quantization Within the Spectral and Spatial Transforms of Sampled Source Contributions to Geophysical Scalar Potential Fields and Its Significance for Education, Research, and Resource Exploration
}

\author{
Andrew M. McDermott ${ }^{1}$ \& Jeffrey R. Chiarenzelli ${ }^{2}$ \\ ${ }^{1}$ Independent research consultant (Physics, Mathematics, Computer Technology), Potsdam, NY, USA \\ ${ }^{2}$ Department of Geology, St Lawrence University, Canton, NY, USA \\ Correspondence: Andrew M. McDermott, Independent research consultant (Physics, Mathematics, Computer \\ Technology), P.O. Box 281, Potsdam, NY, USA. Tel: 1-315-566-1450. E-mail: selkienadiasdad@care2.com
}

$\begin{array}{lr}\text { Received: May 19, } 2013 & \text { Accepted: June 17, } 2013 \quad \text { Online Published: July 11, } 2013 \\ \text { doi:10.5539/mas.v7n8p31 } & \text { URL: http://dx.doi.org/10.5539/mas.v7n8p31 }\end{array}$

\begin{abstract}
Conventional application of fast Fourier transform (FFT) methods in the spectral separation and analysis of surface gravity and magnetization measurements has evolved from the traditional perspective that a continuous spectrum of source contributions overlaps an associated range of wave numbers (frequencies). The FFT algorithm is array-based and the interpolated measurements can be decomposed into a unique and complete set of discrete and disjoint matrices representing vertical components which contribute to the values at each element in the original surface array. The scaling and superposition properties of these components can be exploited in order to develop an alternative approach to the spectral separation of the interpolated surface. The approach has been applied to a variety of interpolated surface arrays, each representing a unique geological region and sampling event. A simulated generalized potential distribution has been designed in order to test and refine analytical methods suggested by the approach. The methods rely upon a principle of optimized scaling of the parameters associated with the field survey. The methods exploit the physical characteristics of the associated potential fields and the mathematical properties of the basis set of vertical spectral components and their equivalent spatial arrays. The approach permits the isolation of spectral components contributed by particular sources within well-defined spatial volumes beneath an associated planar sub-region of the original interpolated surface. Analysis based upon estimates of spatial characteristics for sources represented within the array elements contribute to reduced or minimized ambiguity in interpretations concerned with equivalent-source contributions to the original field measurements.
\end{abstract}

Keywords: spectral separation, discrete analysis, scalar potential, residual, sampling theory, interpolation, fast Fourier transform, digital signal processing, surface gravity, surface magnetization

\section{Introduction}

The foundation of pioneering methods used to locate and characterize resource deposits of petroleum and minerals in the early twentieth century involved the measurement and analysis of gravitational and magnetic attraction at the Earth's surface. The methods were founded in classical theories of the physical forces and the mathematical properties of the continuous Fourier transform (CFT). Researchers like Bhattacharrya (1967) developed sophisticated analytical tools related to the characterization of subsurface sources contributing to the attraction at the surface based upon these principles. These tools provided for the estimation of values related to the depth, size, and density or magnetization of these sources (cf. Sharma, 1997). They were quickly adapted to take advantage of the speed and power of the fast Fourier transform or FFT (cf. Cooley \& Tukey, 1965; Marks, 2009) and related emerging digital signal processing (DSP) technologies. The application of these technologies required understanding the quantitative limitations imposed on the possible resolution of source contributions within the sampled measurements. Gibbs phenomena (Wilbraham, 1848; Gibbs, 1899) in the form of localized aliasing and longer range edge effects are accommodated in the analysis through adapting the results of sampling theory (Shannon, 1945) to the analysis of the geologic datum. These distortions can be associated with the application of the three dimensional FFT to grid-based interpolations of field sampled locations which have been corrected to represent the attraction over a reference plane. The quantitative limitations which affect standard 
analytical spatial and spectral tools have inspired the design of alternative technologies, e.g. discrete wavelet analysis (Hornby et al., 1999) and methods based on finite element concepts (Mallik \& Sharma, 1997). These developments have provided for increasingly sophisticated analysis of subsurface data; however, numerous limitations still exist.

The conventional methods and tools for the acquisition and analysis of measurement of the surface potential have been shaped by the continuous theories and integral equations of traditional geophysics. Datum are treated as components of a continuous surface which must be estimated or generated through modeling and approximation methods. Field surveys are designed in terms of the source bodies of interest to a particular investigation. The samplings are performed at locations which provide an effective average coverage with separations that are a few percent of the survey area of interest (cf. Hinze, 1990; Mishra \& Tiwari, 2011). The information related to smaller, presumably shallow, sources is treated as noise and generally removed or smoothed so as to "...not try to interpolate the data, but rather find an approximation by fitting the error envelope" (Rauth, 1998). The adaptation of conventional analytical methods, as listed previously, requires "precise information about density, depth position, and depth extent of the sources, which cannot be obtained from the ... anomaly alone because of non-uniqueness and stability problems. Quantitative interpretations are, therefore, ill-posed problems. Transforming them into well-posed problems requires additional a priori information.' (Beltrão \& Silva, 1993). The limitations of conventional separation and transformation techniques are also barriers to conventional analysis. These include the requirement for subjective estimates of the properties of presumed sources and the inclusion of carefully selected a priori geological information. Forward modeling and inversion methods, with their associated theoretical problems of non-uniqueness and instability, can be combined with separation techniques in hopes of reducing the non-uniqueness of a solution (Li \& Oldenburg, 1998). These procedures all rely upon the definition of a 'residual anomaly' of interest. As discussed by Hinze (1990) "The definition of the residual anomaly is not very precise ... is problem dependent ... The subjectivity of the residual determination process makes it potentially one of the major limitations of applying gravity and magnetic methods." The analyses and definitions have evolved within the traditional geophysical perspective which regards the scalar potential measurements as representing a continuous functional form.

The full conversion of the theories and tools of Geophysics from analog to digital representation has never been accomplished within the work represented in the relevant literature. The quantized nature of the information related to the unique and disjoint vertical spectral components of the transformed digital surface has never been characterized. The inverse transformation of these components provides access to an equivalent depth-based spatial basis set. This set contains information which can be correlated to the spatial properties of subsurface sources responsible for the measured attractions. An understanding of the characteristics of these fundamental distinct components can be used to improve the efficiency and effectiveness of any separation or enhancement effort directed at an appropriate digital surface (i.e. target or depth of interest).

The purpose in this work is to describe both the methods and the consequences which were a result of efforts to maximize the interpretation and usefulness of subsurface data and optimize survey protocols. The efforts originated with comparisons of the residuals extracted through the systematic application of FFT filters to surfaces interpolated from more than a dozen ground-based gravity and magnetic surveys. These covered areas which represent a broad range of extents and sampling geometries. This analysis was initially performed as an exercise in signal processing techniques, without regard for the geologic context or meaning of the datum. The primary concern was the influence of the available free parameters upon the final filtered signals. The discrete nature of the vertical (depth) information was revealed directly as a consequence of these considerations. Isolation and comparison of these spectral components revealed scaling relationships associated with the vertical spacing between successive components in both the spectral and spatial representations. A specialized simulation was designed in order to distinguish among the possible influences which might determine these scaling relationships. Consideration of the results for both field measured and simulated surfaces served as the basis for generalized, integrated analytical techniques which can be applied to any scalar potential surface interpolated from ground or aerial surveys of the near-surface attraction. They constitute a scaled and optimized method for isolation of the spectral components of any visible influence in the original interpolated surface or any properly filtered residual within a well-defined spatial volume. This volume can be analyzed using conventional methods to extract estimates of the spatial and physical properties of collective or individual source contributions represented within the associated area of the original interpolated surface. The forward models and inversions based upon estimates developed from the described method should be more stable, more reliable, and should lead to less ambiguity in the final interpretations. The reduced technical complexity of the method as compared to conventional approaches to separation of spectral or spatial source contributions to the measured attractions 
make it accessible to all those involved in related educational or research programs. It reduces or eliminates the need for expensive technologies, extensive training and experience, and sophisticated theory-based analytical tools.

The discussion begins with a description of an approach for isolating and identifying the discrete vertical spectral/spatial components. The isolation procedure follows directly from the mathematical form of the FFT algorithm. The application of the method to real and simulated datum leads to a discussion of the implications of the results for analysis, modeling and interpretation. The convention of referring to the coefficients of the FFT array, the frequency coefficients, as 'wave numbers' has been adopted in order to indicate the inverse distance nature of the frequencies. The equivalence of the spectral and spatial information for both isolated and superposed components can be exploited to confine attention primarily to the spatial, or IFFT, representations. This has the advantage of limiting the discussion to real valued arrays whose visual representations have a direct spatial correlation, i.e. they are more likely to be accessible and meaningful to those with limited experience in spectral analysis. The mathematical principles and physical properties that serve as the foundation for this discussion are standard content in all college-level science curricula. The particular integration of the material applied here has no appropriate parallel within the literature. It would be difficult to include details on all the preliminary work without a loss of consistency and clarity. The work was performed using the set of freely available command line tools 'GMT' (http://gmt.soest.hawaii.edu) automated using a simple scripts written in perl (http://www.perl.org). The software provides for the creation of scaled images from the resulting grid files (formatted and georeferenced binary arrays), and the assignment of appropriate visual color gradients and contours. The software is cross-platform by design, and performs efficiently with minimal hardware requirements. Appropriate reference to an extended discussion of the basic principles within the preliminary work and examples of simple scripts has been provided where it is appropriate.

\section{Methods}

\subsection{Formalism}

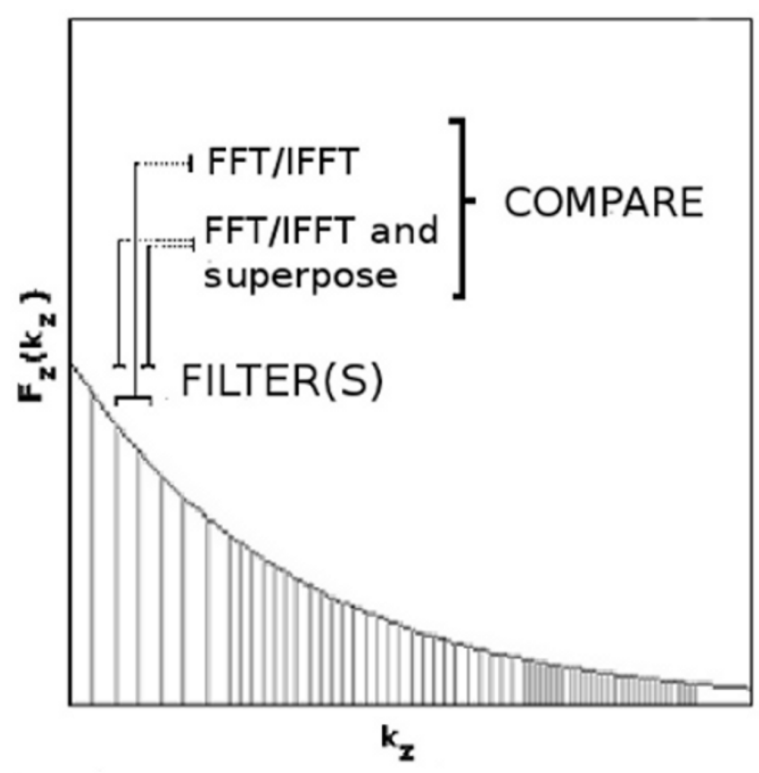

Figure 1. Discrete vertical FFT wavenumber distribution compared to continuous FFT wavenumber distribution with schematic of method for isolating and comparing individual and composite vertical wavenumber components or spatial transform depth components

The components of the FFT can be represented visually as a distribution of discrete Fourier amplitudes $F_{z}\left(k_{z}\right)$ associated with the depth (or $\mathrm{z}$ ) components of the wave numbers $\mathrm{k}_{\mathrm{z}}$ (Figure 1). The three dimensional matrix formalism of the FFT requires a point-by-point nested expansion of complex sinusoidal terms in both directions of the transformation for the vertical transforms, forward $\mathbf{F}(\mathrm{a})$ and inverse $\mathbf{F}^{-1}(\mathrm{~A})$ (adapted from Press et al., 1982):

$$
F\left(a_{i j k}\right) \equiv \sum_{k_{x}=0}^{L-1} \sum_{k_{y}=0}^{M-1} \sum_{k_{z}=0}^{N-1}\left[\exp \left(2 i k_{z}(k / N)\right) \exp \left(2 i k_{y}(j / M)\right) \exp \left(2 i k_{x}(i / L)\right)\right] a_{k x k y k z}
$$




$$
F^{-1}\left(A_{i j k}\right) \equiv(1 / L M N) \sum_{k_{x}=0}^{L-1} \sum_{k_{y}=0}^{M-1} \sum_{k_{z}=0}^{N-1}\left[\exp \left(-2 i k_{z}(k / N)\right) \exp \left(-2 i k_{y}(j / M)\right) \exp \left(-2 i k_{x}(i / L)\right)\right] A_{k x k y k z}
$$

where $\mathrm{a}$ is the spatial surface array indexed by $(\mathrm{i}, \mathrm{j}, \mathrm{k})$ whose $\mathrm{x}, \mathrm{y}, \mathrm{z}$ dimensions are $\mathrm{L}$ by $\mathrm{M}$ by N, A is the complex valued spectral array whose elements are indexed by $\left(\mathrm{k}_{\mathrm{x}}, \mathrm{k}_{\mathrm{y}}, \mathrm{k}_{\mathrm{z}}\right)$, and $2 \mathrm{i}$ denotes twice the square root of -1 . Both sets of indices span the same ranges, from zero to one less than the dimensional count for the respective coordinate.

Software tools often provide facilities for fast Fourier transformations and spectral analysis. Specifically, they allow for the application of spectral filters based upon wave number ranges in the various coordinate directions. The procedures multiply the terms containing wave numbers to be excluded by zero. The finite wave number range available for discrete potential analysis is bounded below by the wave number associated with the record length and above by that of the grid interval representing the sampling length in the plane. The wave numbers are band limited. A consequence of this is that all filters (low/high/band pass) are essentially band pass. The process can be restricted to filters applied with sharp cutoffs, no tapering or buffering, and applied only the vertical direction. The application of a depth-based filtering on any discrete potential surface as described by the transformation equations above becomes a superposition of a subset of individual terms in the Fourier transform. The array elements for any harmonic of the fundamental wavenumber can be retrieved by choosing a fractional wave number increment for the spectral filtering limits. The spatial filtering limits are therefore intermediate to the depths between any two successive vertical components. The sum representing the $n^{\text {th }}$ element of the component array is therefore isolated from the original array elements

$$
F\left(a_{i j k}\right)_{k_{n}-.5}^{k_{n}+.5} \equiv \exp \left(2 i k_{n}(k / N)\right) \sum_{k_{x}=0}^{L-1} \sum_{k_{y}=0}^{M-1}\left[\exp \left(2 i k_{y}(j / M)\right) \exp \left(2 i k_{x}(i / L)\right)\right] a_{k x k y k z}
$$

The effect is equivalent to employing a delta function, where the vertical $\mathrm{k}_{\mathrm{n}} \pm .5$ indicates a filtering operation with sharp cutoffs in the spectral domain. An expression equivalent to equation 3 provides the corresponding vertical spatial residual, or fundamental component elements, for the depth $d_{n}$, i.e. $F^{-1}\left(F\left(a_{i j k}\right)\right)_{n}$. The filter limits can be expressed more intuitively as an interval over the corresponding vertical spatial length scales, or depths, associated with the wave numbers. The full set of ' $\mathrm{N}$ ' such arrays comprise a basis set for the original array in the vertical direction, i.e. a mutually exclusive and exhaustive set of unique component arrays whose elements sum to the original unique composite array. The properties of the FFT and the basic principles of linear algebra guarantee that it is possible to

- filter the terms one at a time with non-overlapping sub-ranges, superpose the spectral transforms and (if desired) inverse transform the individual spectral components and superpose them into a spatial residual

- filter the terms in a single pass with an inclusive range of wave numbers and (if desired) inverse transform this array into a spatial residual

without altering the point by point values of the either the full spectral or spatial representation of the residual. The software in use can be tested by exploiting the exclusive and exhaustive nature of these components as illustrated schematically in Figure 1.

Equation 3 provides the template for an approach which can accomplish this isolation using FFT filter separation of a vertical interval into equal half-ranges. The iterative application of this method within each successive pair of sub-intervals is guaranteed to eventually result in one sub-interval with zero FFT coefficients and one with some non-zero FFT coefficients. This iteration continues within the non-zero half interval until the limit of precision is reached and the filtering fails, i.e. 'throws an exception'. Persistence and organization will reveal all the components within any specified partial (or complete) spectral/spatial interval. The method can be made more efficient and less time consuming once the scaling which characterizes the components is understood.

There is no theory of the FFT. It is an algorithm which implements the infinite and discrete Fourier transform when a fundamental premise (infinite periodicity) has been violated. The components are uniquely determined only after the application of the algorithm to a particular data set. In practice our concern will generally be limited to superposition (linear addition of arrays) for a set of consecutive components. This means a general understanding of the 'rules' will be enough for most analysis. The individual components associated with any depth can be extracted as necessary once a more convenient and efficient procedure can be designed based upon that understanding.

\subsection{Simulations}

Divorcing the digital surface from its geological and physical meaning was the first step towards exploring the 
vertical quantization of the FFT. It was necessary to look beyond real surveys in order to understand the relationship between any particular characteristics of the vertical components which may be revealed by isolating them, along with the various possible influences which may contribute to these, listed below.

- $\quad$ The scaling and other mathematical properties of the Fourier transform

- The choices made in the original sampling geometry in dealing with topographical or logistical constraints, and the combination of higher and lower order resampling (smaller and larger spacing respectively) required to interpolate the irregular datum to a regular grid at a representative average spacing

- The asymmetry in spacing of the traverse points and the distance between traverses in aerial sampling, and the subsequent higher and lower order resampling required to interpolate the traverses to an effective average spacing on a regular grid

- $\quad$ The physical properties of the inverse square attractive force being measured

- $\quad$ The particular source distribution as represented within the interpolated measurements

Distinguishing among these influences required an innovative approach to simulating a digital surface. The surface needed to possess all the necessary mathematical properties without being tied to any particular physical model or set of real geological sources. The computations of necessity were required to be inexpensive, efficient, and easily scripted. The simulation which resulted from all these considerations can be thought of as an enhanced three dimensional extension of an idea presented by Cordell and Grauch (1982) in their discussion of the differences between the continuous and the discrete Fourier transform in one dimension. This approach to numerical simulation of the influence of a generalized scalar potential source distribution had as its goal the isolation of the components of an appropriate synthetic surface referenced to the same geographic region as some representative real surface. It was necessary that this be done for a range of sampling and resampling protocols in order to resolve the influences listed previously. A representative real surface was produced from the field survey illustrated in Figure 2a. The points represent the measured locations of a ground survey conducted and corrected using conventional methods (Revetta $\&$ McDermott, 2003) for a $1^{\circ}$ square region in upstate New York. These points were interpolated at the effective average spacing of $.01^{\circ}$, approximately $1 \mathrm{~km}$ at the latitude and longitude of the survey. The resultant digital surface is shown in Figure $2 \mathrm{~b}$. The contouring and gradient methods used for all surfaces are discussed briefly in the supplemental discussion (McDermott \& Chiarenzelli, 2013). Similar surfaces were also produced by resampling the grid to both $.001^{\circ}$ and $.0001^{\circ}$ spacing. This last spacing involves $10001 \times 10001$ array elements, the practical limit of the available computer resources. There are questions related to the conventional methods of interpolation which required validation of the reliability of resampled information. These questions are addressed by studies performed with the simulation, to be discussed in the following section.

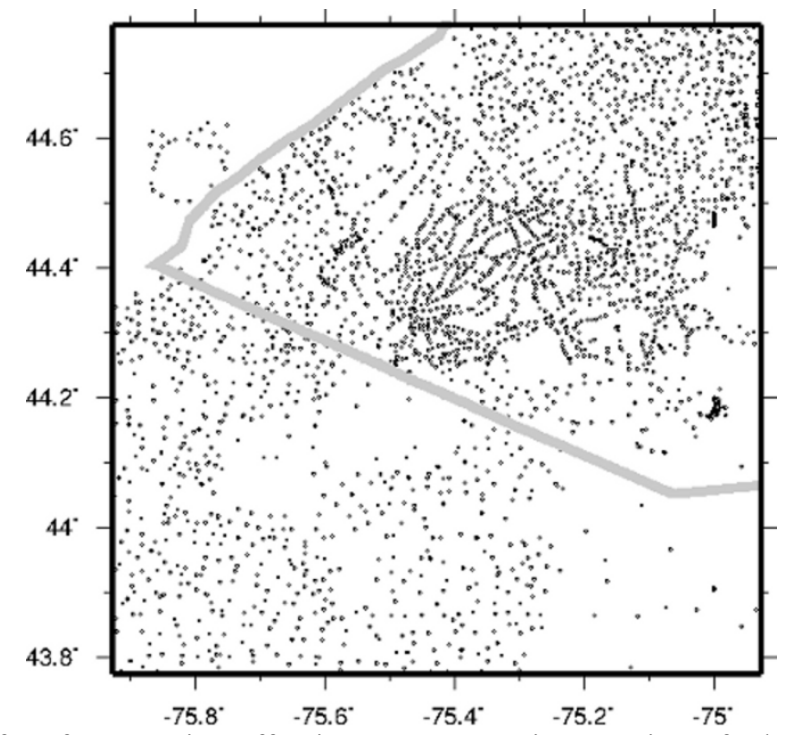

Figure 2a. Field survey of surface gravity, effective average station spacing of $1 \mathrm{~km}$ taken over a $1^{\circ}$ region in upstate New York, St Lawrence county boundary in grey 


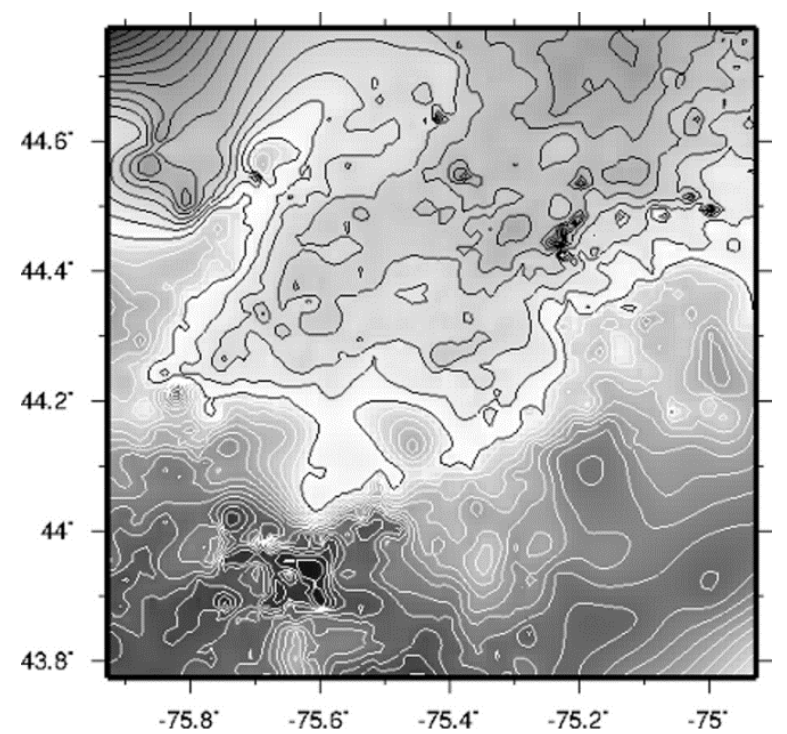

Figure $2 \mathrm{~b}$. Simple Bouguer Anomaly interpolated to a regular grid at $1 \%\left(.01^{\circ}\right)$ spacing over $1^{\circ}$ region from survey of Figure 2a, absolute visual gradient and contour scales applied as described in Appendix, Part1

\subsubsection{Simulation Design}

The simple closed solution for the attraction of the uniform density rectangular prism (Blakely, 1996, Appendix B.6) combined with the mathematical properties of a carefully scaled three level arrangement of symmetrically and periodically spaced sources satisfied all of our requirements. The prisms could be scaled to represent sources of any spatial dimension and physical density or magnetization. The calculations involving a set of prisms scale exactly with the number of prisms in the assembly. The considered use of periodically located and properly scaled source bodies in the plane within layers over scaled depths provides for a dense wave number spectrum which exhibits maximal spectral interference and overlap. The symmetry in the arrangement combined with the periodic reinforcement of contours at all wave number scales guarantees a background against which anomalous effects and computational distortions can be readily detected over any meaningful visible scale of view. The algorithm's point-by-point nature was readily adapted to sampling over the plane in any regular or irregular pattern at any effective spacing within the limits of the available computational memory, storage capacity, and speed. The approach can be implemented in any coding system on a contemporary desktop computer with a minimum of effort. Details concerning the design and implementation of the actual simulated spatial density distribution used are available (McDermott \& Chiarenzelli, 2013).

\subsubsection{Simulations and Higher Order Resampling}

Reports of conventional scalar potential studies in Geophysics generally interpolate the field samples to a grid spacing on the order of the original datum. Finer scale information below this spacing is smoothed or removed, as described briefly in the Introduction. Such studies do not take advantage of the information which may be available within higher order resampling of grid-based datum. This resampling also serves to reduce the possibility of aliasing, resulting in a scaled increase in the spatial resolution available to the analysis. The resampling approach is standard in many other scientific disciplines. Seismologists depend upon multi-dimensional interpolation methods to compensate for irregular or incomplete spatial sampling (cf. Naghizadeh \& Sacchi, 2009). Geologists and geographers in other fields also recognize the value and significance of the information provided through higher order interpolation of georeferenced measurements (cf. Mohd Hasmadi Ismail \& Taylor, 2009). The symmetry and periodicity of the simulation described above provide the means to directly investigate the role of higher order resampling in scalar potential analysis.

The periodicity and symmetry of the contributions within the simulated measurements are reflected, without distortion, in any surface which is interpolated at the original spacing used in the calculation of the array values. The simulated surface sampled at a regular spacing of $.01^{\circ}$ and interpolated with no additional resampling is illustrated by Figure 3a. 


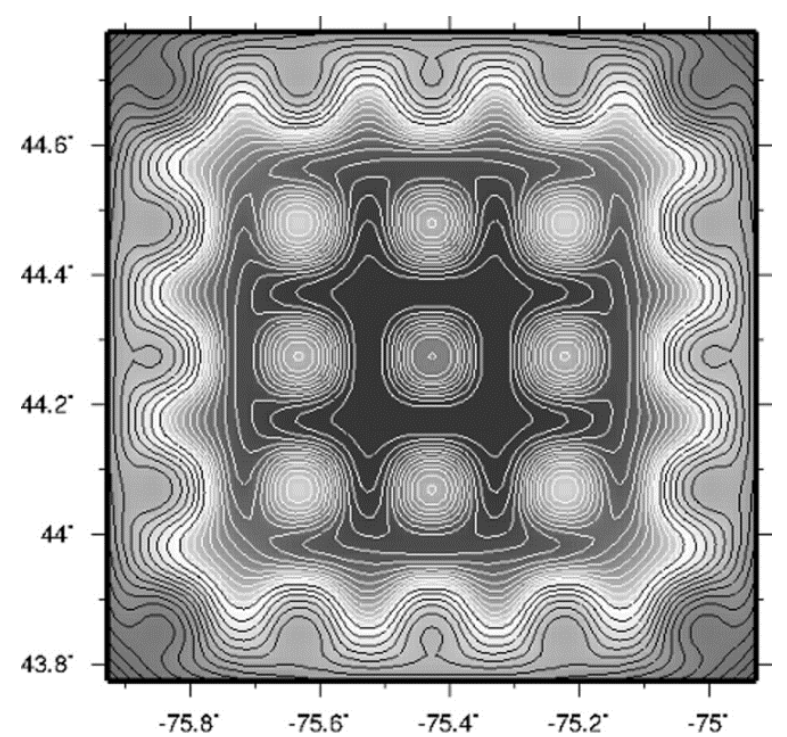

Figure 3a. Simulated 3 layer periodic and symmetric potential, $1 \%$ sampling interpolated to $.01^{\circ}$ regular grid, absolute visual scales applied

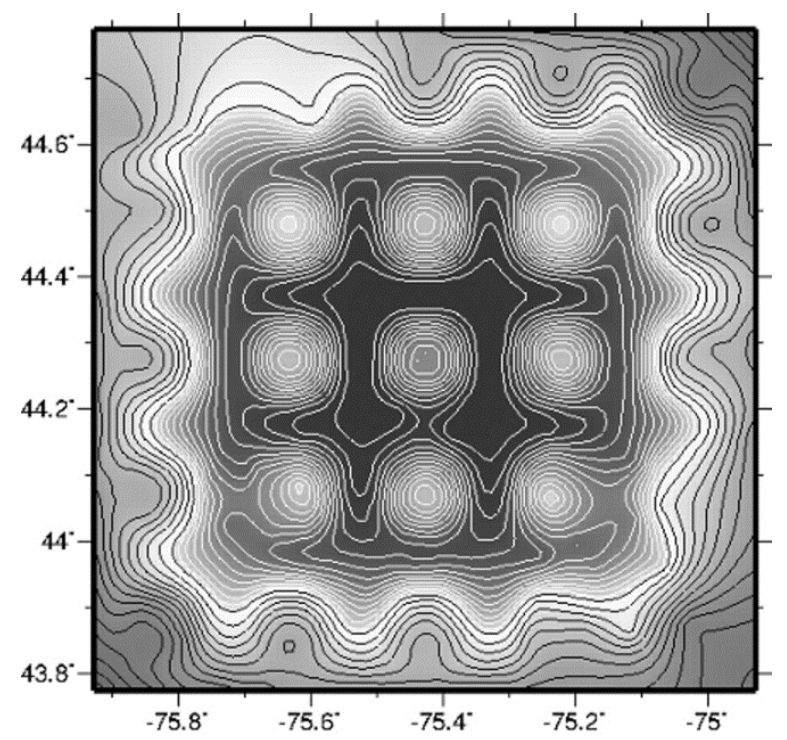

Figure 3b. Simulated potential from sources of Figure 3a sampled according to station locations as indicated for field survey of Figure 2a, interpolated to a $1 \%\left(.01^{\circ}\right)$ regular grid, absolute visual scales applied

Supplemental discussion in McDermott and Chiarenzelli (2013) provides a review of efforts in resampling regular simulations to higher order (smaller) spacing scaled by powers of 10 . The discussion also considers a surface based upon a sampling which simulates the locations of the original field survey mentioned previously over the geographic region of interest and interpolated to $.01^{\circ}$ (Figure $3 \mathrm{~b}$ ). A simulation was generated at the three regular spacings of $.01^{\circ}, .001^{\circ}$, and $.0001^{\circ}$. Each such array was also resampled to represent all other higher order (smaller spacing) grids. The use of a simple array difference technique (a grid summation) permitted direct comparison of the point-by-point variations between a surface whose initial interpolation was resampled to a higher order and a surface sampled and interpolated at that spacing. The evolution of contours and gradient scales within such pairs or sets of surfaces were visually compared. These procedures have been repeated for interpolation methods representing nearest neighbor, inverse distance weighted, and cubic splines with tension algorithms. The results can be summarized as follows:

- There is no evidence of distortion in any significant information at any reasonable scaled view for any surface when regular grids of potential data are resampled to smaller grid spacing over orders of magnitude. 
- Higher order resampling with a well characterized algorithm which honors the characteristics of the potential responsible for the measurements produces consistent contours with an accuracy intermediate to the initial and final spacing at each step as compared to samples taken at the target spacing.

- $\quad$ Resampling to more than 2 higher orders with single precision datum and tools will produce no new useful information.

- There is no true distortion in higher order interpolations, see higher order resampling above, except that noted in the next item. All other apparent distortion is a consequence of the inherent bias of irregularities in the local sampling. This must be accepted or corrected through supplemental field measurements.

- $\quad$ The highest wave numbers corresponding to the smallest length scales in the second order resampling to smaller spacing are associated with interpolation artifacts. They indicate that the valid information has been exceeded. These appear as localized aliasing centered on the grid points associated with the original interpolated measurements. They are visible at highly scaled views of the resampled surface over very small regions. They can be separated entirely from the valid information through the considered application of filtering techniques.

This last point is demonstrated later in the discussion of application of the principles of analysis.

\subsubsection{Comparisons to Field Datum}

It is now necessary to compare the characteristics of the fundamental spatial transforms (IFFT) corresponding to the quantized vertical spectral components (FFT). These must be considered for both of the $1^{\circ}$ regional surfaces (simulated and field sampled) at all samplings, initial interpolations, and final resampled spacing. The entire analysis must also be repeated for surfaces prepared by extracting centrally located square regions representing more localized surveys from every $1^{\circ}$ regional surface, both field measured and simulated. This must be repeated for every equivalent final grid interpolation produced. Regions were chosen with $.1^{\circ}$ and $.12^{\circ}$ sides to provide information concerning the effect of regional extent on the resultant vertical components. An equivalent analysis must be performed for corresponding interpolations of the original field datum

\section{Results}

\subsection{Vertical Spectral Resolution}

The results of the isolation analysis for the depths in meters equivalent to the 50 lowest wave number components are listed by region size and interpolation level in Table 1. The average change in component spacing as a function of depth can be derived from the results listed in Table 2, also arranged by region size and grid spacing. Each column of Table 1 or row of Table 2 is identical for either surface type, real or simulated, for any relevant initial interpolation. 
Table 1. Depths of components vs. region size and sample spacing

\begin{tabular}{|c|c|c|c|c|c|c|}
\hline Region : grid & $1^{\circ}: .01^{\mathrm{o}}$ & $1^{\mathrm{o}}: .001^{\mathrm{o}}, .0001^{\mathrm{o}}$ & $.1^{\circ}: .01^{\mathrm{o}}$ & $.1^{\mathrm{o}}: .001^{\mathrm{o}}, .0001^{\mathrm{o}}$ & $.12^{\circ}: .01^{\circ}$ & $.12^{\circ}: .001^{\circ}, .0001^{\circ}$ \\
\hline \multirow[t]{50}{*}{ Depth (m) } & 142330 & 113864 & 13344 & 10192 & 17792 & 14233 \\
\hline & 101912 & 81529 & 9555 & 8287 & 12739 & 10192 \\
\hline & 82861 & 66289 & 7769 & 7117 & 10358 & 8287 \\
\hline & 71165 & 56932 & 6672 & 5835 & 8896 & 7117 \\
\hline & 58348 & 46678 & 5471 & 5096 & 7294 & 5835 \\
\hline & 50956 & 40765 & 4778 & 4798 & 6370 & 5096 \\
\hline & 47974 & 38380 & 4498 & 4745 & 5997 & 4798 \\
\hline & 47444 & 37955 & 4448 & 4302 & 5931 & 4745 \\
\hline & 43011 & 34409 & 4033 & 4144 & 5377 & 4302 \\
\hline & 41431 & 33145 & 3885 & 3559 & 5179 & 4144 \\
\hline & 35583 & 28466 & 3336 & 3473 & 4448 & 3559 \\
\hline & 34723 & 27779 & 3256 & 3398 & 4341 & 3473 \\
\hline & 33971 & 27177 & 3185 & 3360 & 4247 & 3398 \\
\hline & 33594 & 26875 & 3150 & 3305 & 4200 & 3360 \\
\hline & 33043 & 26434 & 3098 & 3066 & 4131 & 3305 \\
\hline & 30657 & 24526 & 2875 & 2918 & 3833 & 3066 \\
\hline & 29174 & 23339 & 2736 & 2847 & 3647 & 2918 \\
\hline & 28466 & 22773 & 2669 & 2763 & 3559 & 2847 \\
\hline & 27621 & 22097 & 2590 & 2742 & 3453 & 2763 \\
\hline & 27417 & 21934 & 2571 & 2548 & 3428 & 2742 \\
\hline & 25478 & 20383 & 2389 & 2508 & 3185 & 2548 \\
\hline & 25080 & 20064 & 2352 & 2486 & 3135 & 2508 \\
\hline & 24852 & 19881 & 2330 & 2458 & 3107 & 2486 \\
\hline & 24571 & 19657 & 2304 & 2399 & 3072 & 2458 \\
\hline & 23987 & 19190 & 2249 & 2373 & 2999 & 2399 \\
\hline & 23722 & 18978 & 2224 & 2311 & 2966 & 2373 \\
\hline & 23104 & 18484 & 2166 & 2245 & 2888 & 2311 \\
\hline & 22446 & 17957 & 2105 & 2182 & 2806 & 2245 \\
\hline & 21819 & 17455 & 2046 & 2151 & 2728 & 2182 \\
\hline & 21506 & 17205 & 2017 & 2072 & 2689 & 2151 \\
\hline & 20716 & 16573 & 1943 & 2039 & 2590 & 2072 \\
\hline & 20383 & 16306 & 1911 & 2034 & 2548 & 2039 \\
\hline & 20333 & 16267 & 1892 & 2018 & 2542 & 2034 \\
\hline & 20177 & 16142 & 1837 & 1994 & 2523 & 2018 \\
\hline & 19940 & 15952 & 1824 & 1960 & 2493 & 1994 \\
\hline & 19450 & 15676 & 1780 & 1945 & 2450 & 1960 \\
\hline & 18985 & 15560 & 1756 & 1899 & 2432 & 1945 \\
\hline & 18885 & 15188 & 1659 & 1889 & 2374 & 1899 \\
\hline & 18728 & 15108 & 1628 & 1873 & 2361 & 1889 \\
\hline & 17792 & 14982 & 1593 & 1780 & 2341 & 1873 \\
\hline & 17687 & 14233 & 1582 & 1769 & 2224 & 1780 \\
\hline & 17527 & 14149 & 1554 & 1753 & 2211 & 1769 \\
\hline & 17447 & 14021 & 1549 & 1745 & 2191 & 1753 \\
\hline & 17362 & 13958 & 1500 & 1737 & 2181 & 1745 \\
\hline & 16986 & 13890 & 1450 & 1699 & 2171 & 1737 \\
\hline & 16866 & 13589 & 1438 & 1687 & 2124 & 1699 \\
\hline & 16797 & 13493 & 1368 & 1680 & 2109 & 1687 \\
\hline & 16573 & 13438 & 1295 & 1658 & 2100 & 1680 \\
\hline & 16522 & 13258 & & 1653 & 2072 & 1658 \\
\hline & 15992 & 13217 & & 1600 & 2066 & 1653 \\
\hline
\end{tabular}

Table 2. Level parameters at depth by region size

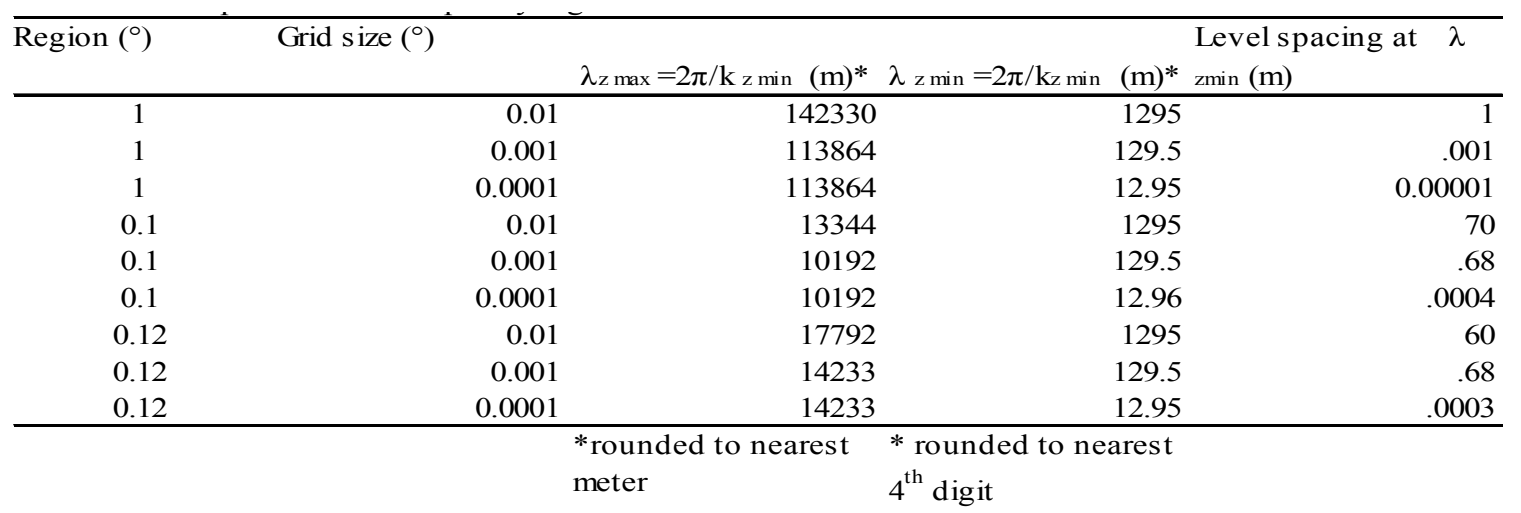


The array which results from superposition, or linear summation, of any set of contiguous (successive) components from the spectrum for a surface must equal the array for the same set of components extracted in the application of a single filter procedure. This is required by the uniqueness properties inherent in the FFT and the potentials involved. Sharply defined filtering limits must be used to prevent mixing or distortion in the elements of individual components. The superposition of the full set of components must equal the original resampled surface array from which they were extracted. This was mentioned previously when discussing the formal equation from which the algorithm is derived. The exclusive and exhaustive nature of the resulting component arrays can be verified directly through array sums and differences. The results of all such procedures for subsets of successive components within any surface have been interpolated, real or simulated, agree within the random error associated with the filtering software.

The results indicate that the spacing of the vertical FFT/IFFT components are determined primarily by the extent, which varies in meters with geographic location for the same extent in degrees, and the regularized spacing of the region. The actual potential surface and/or source distribution of the volume represented by the survey does not seem to matter. The number, wave number/depth, and spacing of components for both the real and simulated surfaces filtered over the same geographic region for $.001^{\circ}$ or $.0001^{\circ}$ spacing $(.01 \%$ and $.001 \%$ of the region dimensions) at all equivalent final interpolations are identical. The same is true, with relevant shifts in the distributions, for surfaces interpolated at $.01^{\circ}(1 \%$ of the region dimensions) with no additional resampling. The resolution, i.e. the number and spacing of components which exist over a particular interval, exhibits a dependence upon the level of interpolation. The accuracy of the vertical spacing seems to be affected adversely by the use of $1 \%$ (or coarser) interpolation over a region, as the components associated with the lowest wave numbers in each surface indicate. These values are significantly larger than would be expected given the known dimensions of the regions. The primary factor which determines the vertical resolution as a function of wave number/depth is the extent of the survey performed. A tenfold increase in the extent of the survey region interpolated to regularized grids increases the available range for vertical components by a factor of ten, with a proportional increase in the average spacing between the additional components. A tenfold decrease in the regularized spacing on that grid provides approximately two additional orders of magnitude of fundamental resolution at higher wave numbers. This is contained within at least one lower order of magnitude of additional depth-based information (toward the surface) for the included source contributions.

\subsection{Vertical Spatial Resolution and Resolution in the Plane}

The superposition of the full set of individual spectral/spatial components returns us to the unique digital signal that is the original interpolated surface. The superposition which characterizes the components of the surface is a consequence of the properties of the simulated potential field. This unique superposition is the only contribution which the physical force makes to the FFT analysis. The values in the surface array, or the values in some contiguous superposed subset, comprise the only absolute reference available for comparisons related to the possible source contributions. These are the last two influences from the original list that must be understood before the physical meaning and geological context represented by the values from the field survey are considered.

The simulation from the previous discussion was used to investigate the manner in which the scaling laws and the vertical superposition combine to provide access to valid depth and planar source information. The methods to be discussed next are contained in McDermott and Chiarenzelli (2013), and were derived from the original considerations related to many distinct gravity and magnetic surveys. The methods are dependent upon the relationships between the vertical components and their spacing as a function of depth and region extent. They incorporate the horizontal scaling of information which results from the higher order sampling. These suggest an approach which can be used to minimize the effects of the Gibbs distortions through the use of visual spatial comparisons of the superposed IFFT components.

Every individual fundamental component is completely contaminated by some combination of aliasing and edge effects. This is determined by combining them through the use of FFT-IFFT filtering processes to represent superposition over varying depths centered about some mean value. The lowest wave numbers at the greatest depths can be combined or decomposed one component at a time. The contributions at shallower depths corresponding to the higher wave numbers are best treated as continuous and should be added and subtracted over multiple components in an interval. The region extent and final grid spacing can be varied for a particular interval and the resultant IFFTs compared. As more components are included over an extended depth interval the central portion of the signal stabilizes and expands from the center. Higher order resampling facilitates this process, and also reduces the aliasing which may remain visible at localized contours within the center. A decrease in edge influence occurs as the size of the region is increased about the center with respect to this stable 
signal. Some of these manipulations produce contours, i.e. visible features, which can be identified in the original interpolation. Other intervals combine to form features which are consistent with those within previously viewed depth intervals which contain them. Eventually the full set of components, either regional or localized within some sub-region, must superpose to form the original interpolation over that particular area. Possible questions concerning the accuracy in the depth associations within the FFT components with respect to real source locations have been resolved in 2 ways: through correlation with existing geologic knowledge of the region associated with the isolated contributions, and through the introduction of properly scaled anomalous bodies at depth in the simulations. The second approach permits comparisons of the FFT residuals from the modified simulation to those from the anomaly simulated and then filtered in an identical manner in isolation.

\section{Discussion}

The consideration of all variations in filtering and superposition leads us back to the context of physical reality and geological validity. It is now possible to discuss methods which can enhance the conventional analysis of scalar potential based digital surfaces. This is accomplished through a scaled increase in the amount of independent quantitative information concerning the relevant sources which can be extracted directly from the field sampled measurements. This corresponds to a scaled decrease in the inherent ambiguity associated with the modeling/inversion, and interpretation stages of an investigation.

\subsection{Three Dimensional Spatial Isolation}

The image in Figure 4 is a representation of separation over depth. It makes use of scaled signal heights for the values in a set of disjoint and complete residual surfaces. These have been extracted from the information of the original field survey interpolated to a $.01^{\circ}$ regular grid as in Figure $2 \mathrm{~b}$. The depth ranges were chosen to correspond to the known geology below this region for the sake of discussion. The Figure 4 represents the IFFTs corresponding to the contributions (from bottom to top) of:

- The nine lowest wave number components associated with depths below the region between $150000 \mathrm{~m}$ and $45000 \mathrm{~m}$ (upper mantle).

- The next six lowest components associated with the crustal transition (Moho) and the lower crust between $45000 \mathrm{~m}$ and $33000 \mathrm{~m}$.

- The 446 superposed components from the intermediate crustal contributions between $33000 \mathrm{~m}$ and $5000 \mathrm{~m}$.

- $\quad$ The highest 1987 wave number components from $5000 \mathrm{~m}$ up to $1295 \mathrm{~m}$.

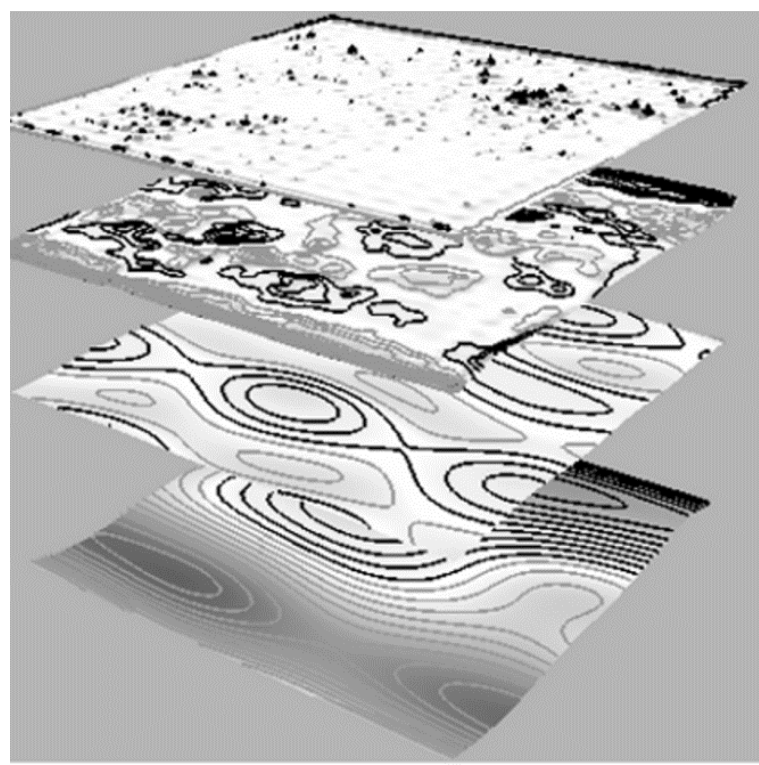

Figure 4. Suggestive proportional depth view of 4 composite residuals, gradient and contours scaled to Figure $2 \mathrm{~b}$, zero level contour omitted from 2 shallowest layers for clarity (GRASS G.I.S. Nviz)

The figures have all been displayed with the gradient scale and contour levels of Figure $2 b$ to give a sense of their relative contributions to the full potential surface. The separation over depth has been accomplished with no 
smoothing of high wave number components, no regional trend removal at the low end of the spectrum, and no synthetic and approximate models for the crustal transition or intermediate components (e.g. decompensative anomalies). The accuracy and resulting resolution inherent in the original information has been preserved at all wave numbers without reduction, mixing, or distortion. The composite layers can be analyzed with power spectrum techniques, gradient or directional filtering, or continuation methods. Possibly, these can be correlated directly to features within the spatial residuals or the original interpolated surface. Additional filtering can be applied within the depth intervals of interest in efforts to improve the available vertical resolution for particular anomalies of interest. Sub-regions representing more localized anomalous contributions can be extracted and examined spectrally and spatially to consider the planar resolution among anomalies at each depth. The sub-regions containing such anomalies can be chosen so as to reduce or eliminate visible edge effects and aliasing. The spectra can be analyzed to verify the freedom from, or degree of, contamination.

The example residuals of Figure 4 and the generalized methods mentioned above represent separations in both vertical and horizontal wave number components which scale over several orders of magnitude for a single choice of region extent and interpolation for the original surface. These separations rely on a minimum of prior characterization of the sources responsible for the measured attractions. Conventional spectral analysis is based in a tradition which relies heavily upon estimates related to a single regional-residual separation between the low and high vertical wave number contributions to the measured potentials. This separation requires a priori subjective assumptions concerning the nature of the source distribution responsible for the measurements as well as the application of subjective reductions (e.g. smoothing) to the interpolated datum. Enhancement methods, e.g. deconvolution, continuation, gradient or directional filtering, and finite element approaches, are based in analytical procedures which also require some assumptions concerning the source distribution. They also mix or alter the information through the implicit assumptions of continuity and essential inseparability.

The utility of the spatial isolation approach to preliminary analysis can be demonstrated through the use of a single example. Target depths are selected within the regional volume of the highest wave number contributions from the field survey with an average spacing of $1 \%$ of the regional dimensions. This is the uppermost residual displayed in Figure 4. The localized features within an area approximately $3 \mathrm{~km}$ on a side at the center of the region are the focus of the examination. The effective sample spacing of the existing survey over this $100 \mathrm{~km}$ region is unsuitable for conventional analysis at these depths of interest for reasons discussed in the Introduction. Figure $5 \mathrm{a}$ is the information from the original surface and within the small central region for the $1 \%$ interpolation. Figure $5 \mathrm{~b}$ is this information for a surface resampled to a $.01 \%$ grid $\left(.0001^{\circ} \mathrm{spacing}\right)$.

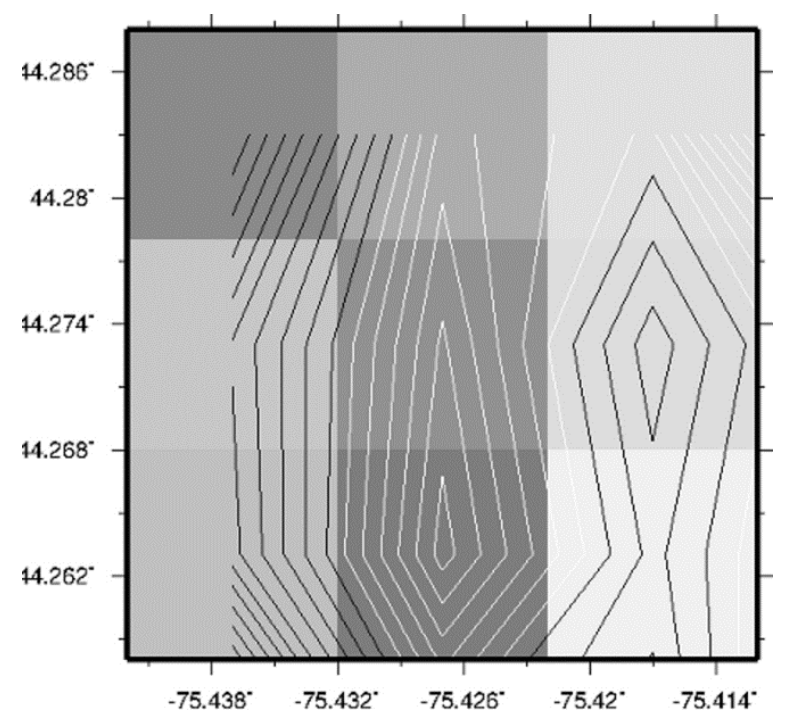

Figure $5 \mathrm{a} . .03^{\circ}$ central region $(\sim 3 \mathrm{~km})$, irregular field data from Figure 2 a extracted using grid commands (array methods) from full survey interpolated at $1 \%\left(.01^{\circ}\right)$ over $1^{\circ}$ regular grid (Figure $2 \mathrm{~b}$ ), absolute gradient and contours 


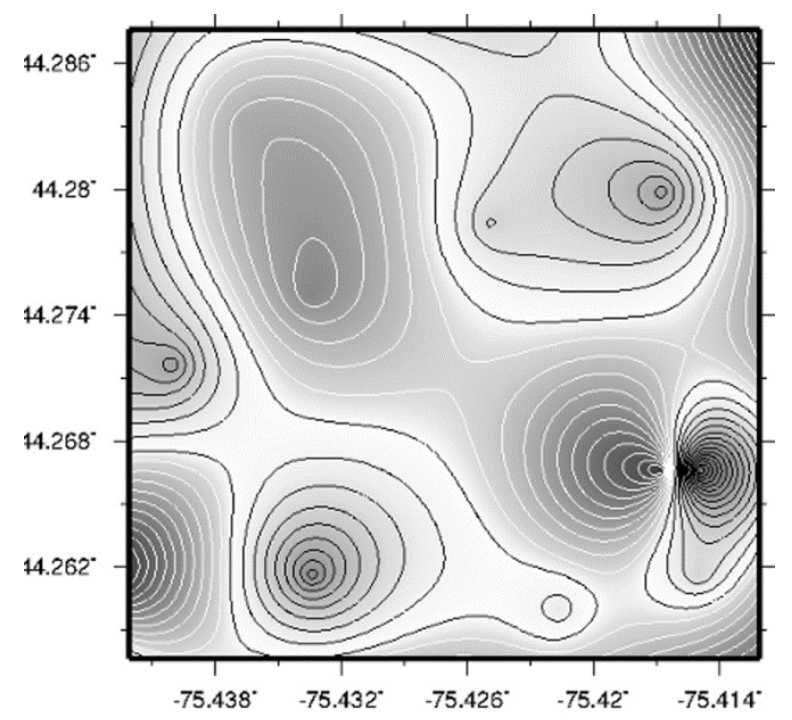

Figure $5 \mathrm{~b} . .03^{\circ}$ central region $(\sim 3 \mathrm{~km})$, irregular field data from Figure 2a extracted using grid commands (array methods) from full survey after resampling to $.01 \%\left(.0001^{\circ}\right)$ over $1^{\circ}$ regular grid (Figure $2 \mathrm{~b}$ ), absolute gradient and contours

Based upon the same historical interest which led to selection of this region, attention for now will remain restricted to the contributions from sources filtered sharply between the depths of $1200 \mathrm{~m}$ and $400 \mathrm{~m}$ below the surface. All figures to be discussed are displayed with an absolute contouring and gradient scale, i.e. to the range of the information in the figure. Figure $6 \mathrm{a}$ is the $3 \mathrm{~km}$ central region when only the information within this $3 \mathrm{~km}$ region for the $.01 \%$ resampled grid is used as the basis for filtering the depth contributions. The visible features are completely obscured by oscillatory contamination. Figure $6 \mathrm{~b}$ is the same small $.01 \%$ grid region filtered over the same depths using a $.12^{\circ}$ central region for the filtering reference. The visible features in this figure have a distinct linearity in both dimensions, indicative of edge effects. Figure 6c repeats the filtering with the full information of the $1^{\circ}$ grid at $.01 \%$ resampled spacing included as the reference surface for the extraction. Figure $6 \mathrm{~d}$ is the full view of the $.12^{\circ}$ surface used for producing Figure $6 \mathrm{~b}$, after the filtering has been performed. It is obvious that the entire residual for this region, even the central $3 \mathrm{~km}$ area, is contaminated by edge effects. Evidence of these effects is especially pronounced along the borders of the visible region. Figure 6e displays the $.12^{\circ}$ region containing Figure $6 \mathrm{c}$ after the full $1^{\circ}$ region filter has been applied. The figure is free of any visual evidence of edge effects or aliasing over this central region when the filtering has been performed using the $1^{\circ}$ region as the reference surface. It is easy to confirm that information within the central $10 \%$ of the reference region, a region about $30 \mathrm{~km}$ on a side, will remain undistorted when the FFT separation is conducted over a region with $1^{\circ}$ extent and $.01 \%$ spacing. The minimum intervals over which such information will remain undistorted average about $10 \%$ of the mean depth of the filter if it is applied over a $1^{\circ}$ reference region interpolated at $.01 \%$. The limit of the method is reached at a depth of approximately half the original effective survey spacing, i.e. $50 \mathrm{~m}$ intervals at $500 \mathrm{~m}$. Scaling considerations based upon simulations and other surveys suggest this resolution could be improved through the use of a regularized field survey characterized by $.1 \%$ or finer coverage. 


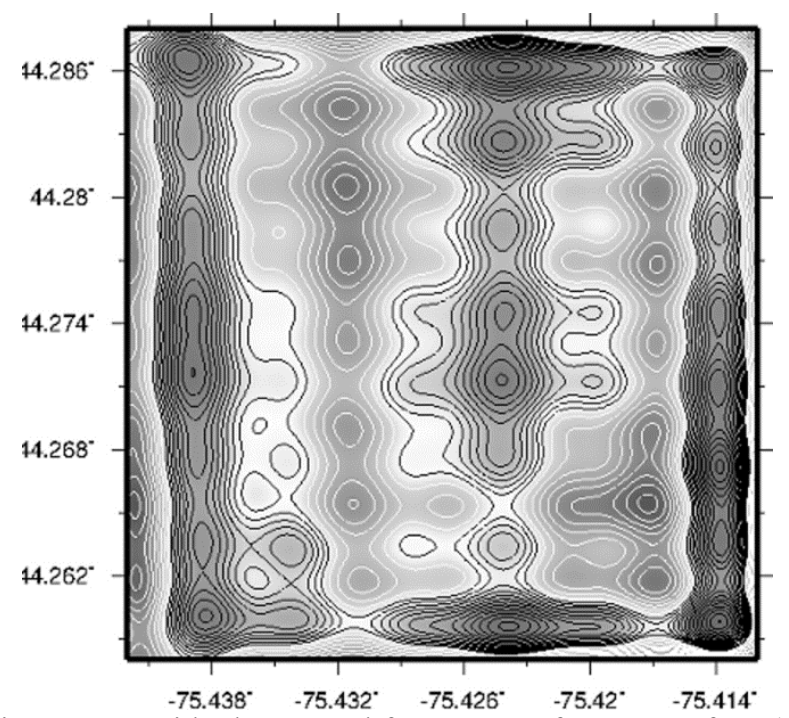

Figure 6 a. $.03^{\circ}$ central region IFFT residual removed from $.03^{\circ}$ reference surface $\left(.0001^{\circ}\right.$ resample) after FFT filter with sharp cut offs applied for depths from $1200 \mathrm{~m}$ to $500 \mathrm{~m}$, absolute gradient and contours with complete oscillatory distortion evident

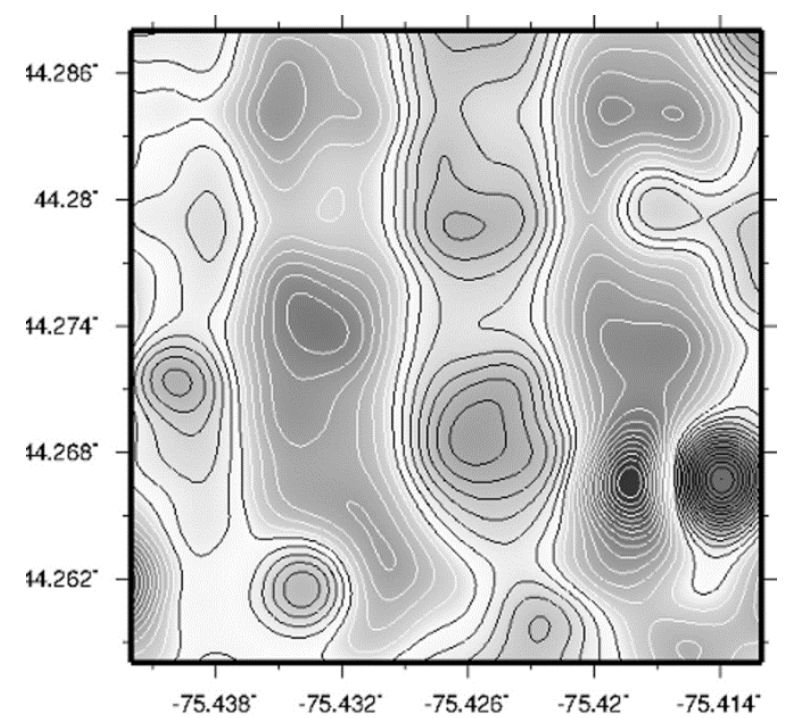

Figure $6 \mathrm{~b} . .03^{\circ}$ central region IFFT residual removed from $.12^{\circ}$ reference surface $\left(.0001^{\circ}\right.$ resample $)$ after FFT filter with sharp cut offs applied for depths from $1200 \mathrm{~m}$ to $500 \mathrm{~m}$, absolute gradient and contours with evidence of strong oscillatory distortion 


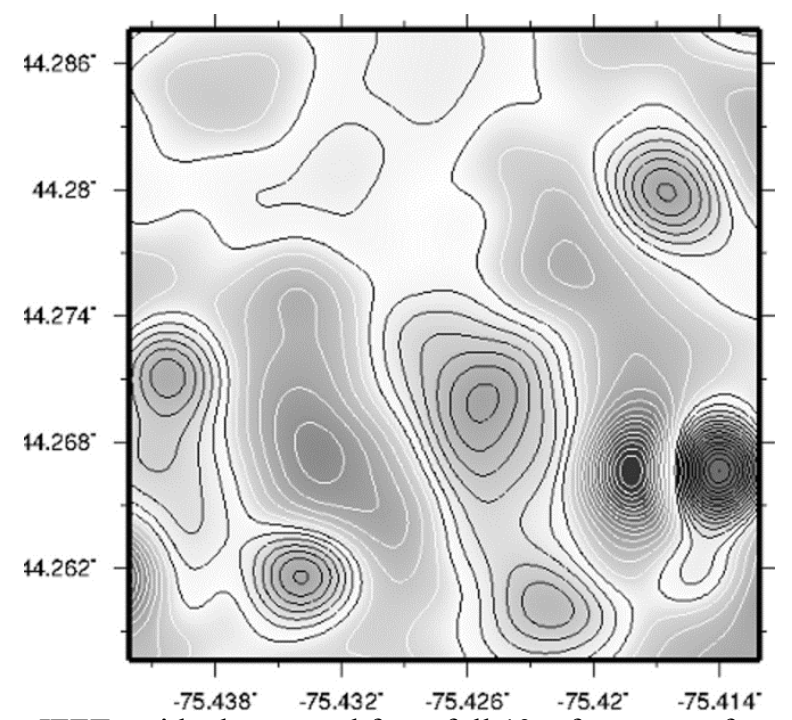

Figure $6 \mathrm{c} . .03^{\circ}$ central region IFFT residual removed from full $1^{\circ}$ reference surface $\left(.0001^{\circ}\right.$ resample) after FFT filter with sharp cut offs applied for depths from $1200 \mathrm{~m}$ to $500 \mathrm{~m}$, absolute gradient and contours free of all distortion

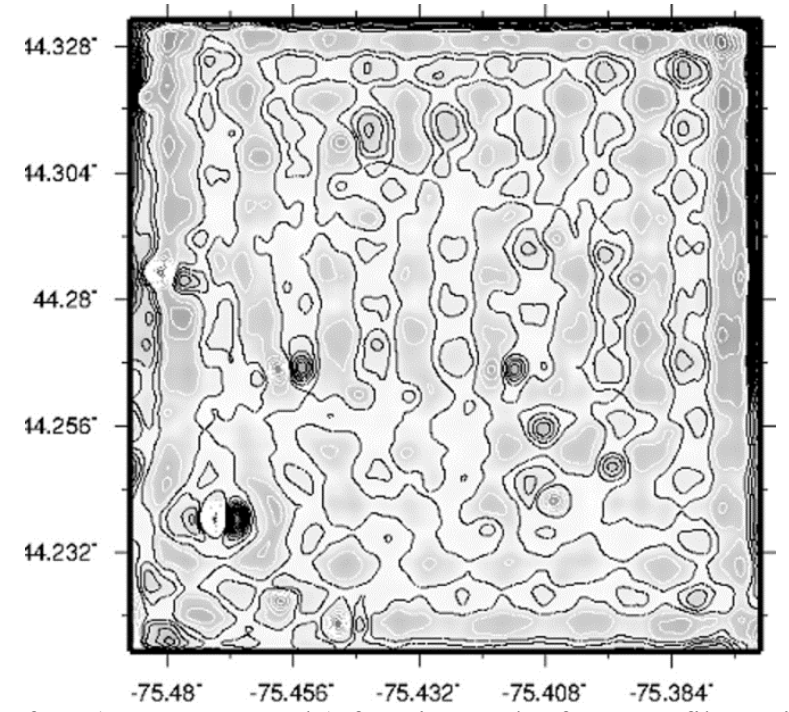

Figure $6 \mathrm{~d} .12^{\circ}$ reference surface $\left(.0001^{\circ}\right.$ resample) for Figure $6 \mathrm{~b}$ after FFT filter with sharp cut offs applied for depths from $1200 \mathrm{~m}$ to $500 \mathrm{~m}$, absolute gradient and contours, oscillatory distortion present across visible region 


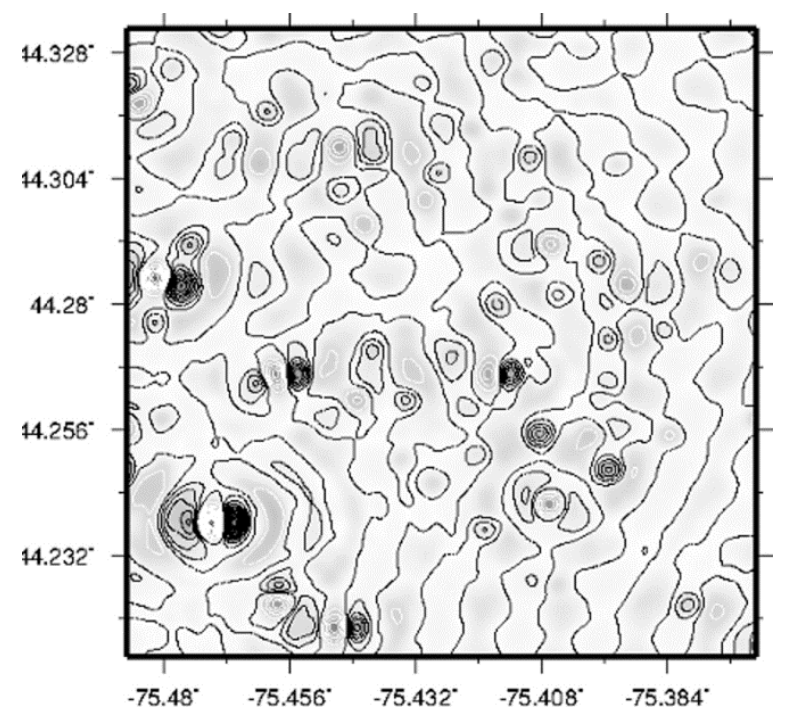

Figure 6e. $.12^{\circ}$ surface $\left(.0001^{\circ}\right.$ resample) containing Figure $6 \mathrm{c}$ after FFT filter with sharp cut offs applied to full $1^{\circ}$ reference surface for depths from $1200 \mathrm{~m}$ to $500 \mathrm{~m}$, absolute gradient and contours, free of distortion within visible region

The contours in the paired negative/positive anomalies to the southwest in Figure $5 \mathrm{~b}$ contain artifacts from the interpolation as described earlier in the discussion. The filter applied to produce Figure $6 \mathrm{c}$ avoids these artifacts. This can be confirmed by extracting the full region residual between depths of $400 \mathrm{~m}$ and the surface $(\mathrm{z}=0)$ for the $.01 \%$ resampled interpolation. It is possible to verify the freedom from edge and alias distortion in Figure $6 \mathrm{c}$ through a combination of the region extent/filter interval/resampling comparisons already described. This is essentially an extension of the process used to produce the Figures 5a through $6 \mathrm{e}$. The information in Figure $6 \mathrm{c}$ can be compared to that of Figure $5 \mathrm{~b}$ to confirm the correlation of the locations visible within the filtered contributions which correspond to significant contributions to the original surface within this small region.

The available supplemental discussion (McDermott \& Chiarenzelli, 2013) describes the estimation of physical properties of isolated source contributions. It applies the uniform vertical density/magnetization approximation to regions extracted as residuals which may be considered to represent scaled single layers of geological information. The method relies on a statistical distribution for the included surface array values binned in a manner which reflects the contour levels of the visual surface representation of the included grid points. The normalized and corrected areas under such a curve correspond to the relative densities/magnetizations for the spatially correlated spatial volumes between the included contours.

\section{Conclusions}

The three dimensional fast Fourier transform of field sampled scalar potential source attractions interpolated to a regular grid results in discrete vertical spectral elements. These serve as a mutually exclusive and exhaustive set of array-based wave number components scaled in the vertical coordinate. The scaling characteristics of the separation between successive components and the superposition properties they inherit from both the FFT and the potential provide for a set of minimum uncertainty spectral filters. These correspond to a set of minimum ambiguity depth components in the spatial domain. Sampling theory predicts the limits on the planar spatial resolution which can be achieved for any grid-based surface. Conventional analytical methods and principles have been adapted based upon an understanding of the implications of these planar and vertical resolution limits. The result is an enhanced method for isolating and separating the influence of distinct source contributions to the original field measurements. The approach is based primarily upon quantitative visual comparisons and simple direct calculations involving FFT filtered residual surfaces.

The methods described form the basis for a scaled and optimized approach to the problem of separating particular spectral contributions and isolating them within well-defined spatial volumes (McDermott, 2006). These can be applied across the full spectrum of contributions in any interpolated scalar potential based surface. At any point in the analysis the information within a particular spatial volume can be compared to existing or assumed geological information, subjected to conventional separation and enhancement techniques, or used directly to supply estimated values for use in modeling and inversion studies. The approach can be useful in reducing the burdens imposed on conventional analysis as a consequence of the reliance on a priori information 
and subjective estimations.

Combined with existing analytical spatial and spectral methods this approach can increase the accuracy and amount of quantitative information available for interpretation by scaled orders of magnitude. This directly reduces the need for preliminary subjective estimates and the inclusion of independent a priori information. The ambiguity which affects the final interpretations associated with conventional modeling and inversion can minimized through the careful and considered use of the isolation techniques. As stated by Götze (2011), "In practical solutions of inversion problems, the direct problem of gravity appears, i.e., the computation of gravitational effects caused by particular mass contributions given in terms of location, shape, and density. In this case, a unique solution is always possible by application of the law of gravitation...". Proper application of the methods, when included within a relevant survey and associated analysis, can reduce or eliminate the need for implementation of any of the conventional spectral or spatial methods of separation and enhancement.

Applied separately the approach may serve as an alternative to those programs which lack the resources or expertise to implement a more conventional analysis. The analytic procedures involve the IFFT, i.e. they are spatial and intuitive. The methods are simple to implement and can be employed with minimal training and experience. They can be applied to any appropriately acquired set of measurements, and can inform any planned study concerning the most effective and efficient survey design. The approach can be adapted to the goals and resources available to any academic or professional investigation. It is based in fundamental physical principles, basic mathematical methods, and resource spare computational techniques which are accessible and available within any standard undergraduate science curriculum. Such methods remove the artificial barriers to understanding and discovery which can be imposed by the resource intensive and technically complex techniques required by conventional analytical techniques.

The approach as suggested provides those involved in gravity and magnetic analysis with resolution and accuracy comparable or superior to other non-invasive exploration tools over an applicable depth range. The direct information regarding possible sources has predictable accuracy at the depths associated with the most desirable target bodies in modern exploration. The techniques favor ground-based survey techniques, the simplest and most resource sparing approach available. The methods can be applied to any existing set of relevant data. They also provide the means to evaluate the most effective approach to designing new surveys or to augment available datum. The reduced technical requirements combined with the increased analytical capacity should lead to more efficient, effective, and sustainable exploration and exploitation.

\section{Acknowledgements}

The Authors' thanks and appreciation to Dr. Frank Revetta for his data and mentoring, William de Lorraine for proposing the original question, and Bob and Joe for their enthusiasm and editorial assistance. The Authors wish to acknowledge the significance of comments and suggestions provided by Dr. Richard Blakely of the U.S.G..S. in review of our preliminary experimental results in 2004.

\section{References}

Beltrão, J. F., \& Silva, J. B. C. (1993). Mapping and depth ordering of residual gravity sources. Geophysics, 58, 1408-1416. http://dx.doi.org/10.1190/1.1443356

Bhattacharrya, B. K. (1967). Some general properties of potential fields in space and frequency domain: a review. Geoexploration, 5, 127-143. http://dx.doi.org/10.1016/0016-7142(67)90021-X

Blakely, R. J. (1996). Potential theory in gravity \& magnetic applications. p. 464. Cambridge UK: Cambridge University Press. http://dx.doi.org/10.1017/CBO9780511549816

Cordell, L., \& Grauch, V. (1982). Reconciliation of the discrete and integral Fourier transforms. Geophysics, 47, 237-243. http://dx.doi.org/10.1190/1.1441330

Cooley, J. W., \& Tukey, J. W. (1965). An algorithm for the machine calculation of complex Fourier series. Math. Comput, 19(90), 297-301. http://dx.doi.org/10.1090/S0025-5718-1965-0178586-1

Gibbs, J. W. (1899). Fourier's Series. Journal on Mathematical Analysis, 15, 723-736.

Götze , H. J. (2011). Encyclopedia of solid earth geophysics edited by Harsh K. Gupta, Dordrecht. The Netherlands: Springer, p. 503.

Hinze, W. J. (1990). The role of gravity and magnetic methods in engineering and environmental studies. Geotechnical and Environmental Geophysics, 1, 75-126.

Hornby, P., Boschetti, F., \& Horowitz, F. G. (1999). Analysis of potential field data in the wavelet domain. 
Geophysical Journal International, 137, 175-196. http://dx.doi.org/10.1046/j.1365-246x.1999.00788.x

Ismail, M. H., \& Taylor, J. C. (2009). Investigation of Differences of Topographical Map and GIS-derived Spatial Map with Actual Ground Data in Peninsular Malaysia. Modern Applied Science, 3(1), 81-88.

Li, Y., \& Oldenburg, D. W. (1998). Separation of regional and residual magnetic field data. Geophysics, 63(2), 431-439. http://dx.doi.org/10.1190/1.1444343

Mallick, K., \& Sharma, K. K. (1997). Computation of regional gravity anomaly-a novel approach. Proceedings of the Indian Academy of Sciences - Earth and Planetary Sciences, 106(1-2), 55-59.

Marks, R. J. (2009). Handbook of Fourier Analysis and its Applications. p. 772. New York: Oxford University Press, Inc.

McDermott, A. M. (2006). Method for enhancing depth and spatial resolution of one and two dimensional residual surfaces derived from scalar potential data, U.S. Patent 7043366 (Canadian Patent 2456459, 2009).

McDermott, A. M., \& Chiarenzelli, J. R. (2013). Supplemental background discussions and sample scripts for Quantization within the spectral and spatial transforms of sampled source contributions to geophysical scalar potential fields and its significance for education, research, and resource exploration, available by request to the authors.

Mishra, D. C., \& Tiwari, V. M. (2011). Encyclopedia of solid earth geophysics edited by Harsh K. Gupta, Dordrecht. p. 513. The Netherlands: Springer.

Naghizadeh, M., \& Sacchi, M. (2009). f-x adaptive seismic-trace interpolation, Geophysics, 74(1), 9-16. http://dx.doi.org/10.1190/1.3008547

Rauth, M., \& Strohmer, T. (1998). Smooth approximation of potential fields from noisy scattered data. Geophysics, 63, 85-94. http://dx.doi.org/10.1190/1.1444330

Revetta, F. A., \& McDermott, A. (2003). The compilation and preparation of high resolution gravity data for petroleum exploration in New York State and adjoining regions. New York State Energy Research Development Authority (NYSERDA) PON \#715-02.

Shannon, C. E. (1949). Communication in the presence of noise. Proc. Institute of Radio Engineers, 37(1), $10-21$.

Sharma, P. V. (1997). Environmental and Engineering Geophysics. p. 475. Cambridge University Press. http://dx.doi.org/10.1017/CBO9781139171168

Press, W. H., Teukolsky, S. A., Vetterling, W. T., \& Flannery, B. P. (1992). Numerical recipes in C: The art of scientific computing ( $2^{\text {nd }}$ ed.). p. 995. Cambridge University Press.

Wilbraham, H. (1848). On a certain periodic function. The Cambridge and Dublin Mathematical Journal, 3, 198-201.

\section{Copyrights}

Copyright for this article is retained by the author(s), with first publication rights granted to the journal.

This is an open-access article distributed under the terms and conditions of the Creative Commons Attribution license (http://creativecommons.org/licenses/by/3.0/). 